\title{
Emodin potentiates the anticancer effect of cisplatin on gallbladder cancer cells through the generation of reactive oxygen species and the inhibition of survivin expression
}

\author{
WEI WANG ${ }^{1}$, YUEPING SUN ${ }^{2}$, XINXING LI ${ }^{1}, \mathrm{HUI} \mathrm{LI}^{2}$, \\ YUYING CHEN $^{2}$, YE TIAN ${ }^{2}$, JING YI $^{2}$ and JIAN WANG ${ }^{1}$ \\ ${ }^{1}$ Division of General Surgery, Renji Hospital, Shanghai Jiao Tong University School of Medicine, \\ 1630 S. Dongfang Road, Shanghai 200127; ${ }^{2}$ Department of Cell Biology, Key Laboratory of the \\ Education Ministry for Cell Differentiation and Apoptosis, Institutes of Medical Sciences, Shanghai Jiao \\ Tong University School of Medicine, 280 S. Chongqing Road, Shanghai 200025, P.R. China
}

Received May 25, 2011; Accepted June 24, 2011

DOI: $10.3892 /$ or.2011.1390

\begin{abstract}
Gallbladder carcinoma is known to be an aggressive malignancy and non-sensitive to routine chemotherapy; its prognosis is quite poor. In this study, we show that emodin (1,3,8-trihydroxy-6-methylanthraquinone), an active component from Chinese medicinal herbs, can enhance apoptosis of gallbladder cancer cells induced by cisplatin (CDDP) in a reactive oxygen species (ROS)-dependent manner. The expression of survivin, which is involved in the inhibition of apoptosis, was measured after drug treatment and it was found that this could be suppressed by CDDP. Co-treatment with emodin additively inhibited survivin expression in a ROS-dependent manner. Further experiments proved that emodin potentiated the antitumor effects of CDDP in vivo by downregulating the expression of survivin without causing detectable toxic effects on normal tissues.
\end{abstract}

\section{Introduction}

Gallbladder cancer is a highly aggressive malignant disease. Radical resection is still the most effective treatment for

Correspondence to: Dr Jian Wang, Division of General Surgery, Renji Hospital, Shanghai Jiao Tong University School of Medicine, 1630 S. Dongfang Road, Shanghai 200127, P.R. China

E-mail: dr_wangjian@yahoo.com.cn

Abbreviations: emodin, 1,3,8-trihydroxy-6-methylanthraquinone; ROS, reactive oxygen species; P-gp, permeability glycoprotein; CDDP, cisplatin; DCFH-DA, 2,7-dichlorodihydrofluorescein diacetate; DCF, 2,7-dichlorofluorescein; MTT, 3-(4,5-dimethylthiazol2-yl)-2,5-diphenyltetrazolium bromide; annexinV-FITC, annexin V-fluorescein isothiocyanate; PI, propidium iodide; IAP, inhibitor of apoptosis protein; HIF-1, hypoxia inducible factor-1; redox, oxidationreduction; $\mathrm{As}_{2} \mathrm{O}_{3}$, arsenic trioxide; $\mathrm{NF}-\kappa \mathrm{B}$, nuclear factor $\kappa \mathrm{B}$; NAC, $\mathrm{N}$-acetyl-cysteine; SSC, standard sodium citrate

Key words: cisplatin, reactive oxygen species, survivin, emodin, gallbladder cancer, chemotherapy, drug resistance gallbladder cancer, however, the overall survival rate remains quite poor (1). Chemotherapy is the most common available treatment for the malignancy. However, gallbladder tumors are known as chemotherapy-insensitive. Many researchers have tried to enhance the chemosensitivity of gallbladder cancer cells either in clinical or in experimental field, however, no satisfactory results have been achieved (2).

Cisplatin (CDDP) is one of the most potent and widely used anti-cancer agents in the treatment of various solid tumors. Insufficient sensitivity or resistance to CDDP is usually the major obstacle for its effective application in cancers including gallbladder cancers. We have previously demonstrated that emodin (1,3,8-trihydroxy-6-methylanthraquinone), a kind of natural anthraquinone enriched in the traditional Chinese herbal medicines, sensitizes Du-145, a cell line derived from prostate carcinoma, to CDDP in ROS-dependent manner (3). In addition, we found that the multidrug resistance protein P-gp (permeability glycoprotein) is downregulated and its transcription factor, hypoxia inducible factor-1 (HIF-1), is inhibited. In the present study we further investigate if emodin can synergize CDDP in gallbladder cancer cells, in order to verify the general effectiveness of emodin. In addition, we explore whether the prosurvival molecule survivin is involved in the effects of emodin.

Survivin is a member of the inhibitor of apoptosis gene family, which is involved in control of cell division and inhibition of apoptosis. This protein, which is expressed in the most common human cancers, exerts its anti-apoptotic activity and chemoresistance by interfering with the processing and activity of caspases $(4,5)$.

In this study, we investigated our hypothesis that emodin combined with CDDP would show a synergistic effect on gallbladder cancer cell proliferation and apoptosis. The results presented in the current study suggest that emodin in combination with CDDP caused greater antitumor activity than CDDP alone in vitro and in vivo, which was correlated with down-regulation of survivin levels mediated by ROS generation. 


\section{Materials and methods}

Cells and reagents. The human gallbladder cancer cell line SGC996 was provided by Academy of Life Sciences, Tongji University (Shanghai, China). SGC996 cells were maintained in RPMI-1640 medium (Gibco BRL, Gaitherburg, MD, USA). The media were supplemented with antibiotics and $10 \%$ newborn calf serum. Cells were cultured in a humidified atmosphere with $5 \% \mathrm{CO}_{2}$ at $37^{\circ} \mathrm{C}$. CDDP was obtained from Qilu Pharmaceutical Co., Ltd. (Ji Nan, China). Emodin and NAC (N-acetyl-cysteine) were all purchased from Sigma (St. Louis, MO, USA).

Cell viability assay. Cells were seeded at $1.5 \times 10^{4} / \mathrm{ml}$ cells per well in 96-microculture-well plates. After exposed to the agents as indicated for $24 \mathrm{~h}$, cell viability was assayed using the 3-(4,5-dimethylthiazol-2-yl)-2,5-diphenyl-tetrazolium bromide (MTT) (Sigma) assay as previously described $(6,7)$.

Cell apoptosis analysis. Cells were treated with drugs for $24 \mathrm{~h}$ and apoptotic rates were assessed with flow cytometry using Annexin V-fluorescein isothiocyarate (Annexin V-FITC)/ propidium iodide (PI) kit (BD Pharmingen, San Diego, CA, USA). Samples were prepared according to the manufacturer's instructions and analyzed by flow cytometry on FACS Calibur (Becton Dickson, San Diego, CA, USA) $(6,7)$.

ROS measurement. 2,7-Dichlorodihydrofluorescein diacetate (DCFH-DA) (Sigma) was used as ROS capture in the cells. The average fluorescent intensity of 2,7-dichlorofluorescein (DCF) stands for intracellular ROS levels $(6,7)$. Cultured cells were exposed to various drugs and $10 \mu \mathrm{M}$ of DCFH-DA at $37^{\circ} \mathrm{C}$ for $15 \mathrm{~min}$, with pre-incubation of NAC for $4 \mathrm{~h}$. After washing once with ice-cold PBS, cells were harvested and kept on ice for an immediate detection by flow cytometer FACS Calibur.

Reverse transcriptase-polymerase chain reaction ( $R T-P C R)$. Expression of the survivin was monitored by RT-PCR. SGC996 cells were lysed with $1 \mathrm{ml}$ of RNAse-clean TRIzol reagent (Invitrogen, Carlsbad, CA, USA) after treatment or not, and then the samples were processed according to the manufacturer's protocol to obtain total cellular RNA. The isolated total RNA $(1 \mu \mathrm{g})$ was reverse-transcribed using random primers and AMV reverse transcriptase (Promega, Madison, WI, USA) for $5 \mathrm{~min}$ at $70^{\circ} \mathrm{C}, 5 \mathrm{~min}$ on ice and $60 \mathrm{~min}$ at $37^{\circ} \mathrm{C}$. The single-stranded cDNA was amplified by polymerase chain reaction using GoTaq DNA polymerase (Promega, Madison, WI, USA). PCR of survivin gene was performed under the following conditions: $30 \mathrm{sec}, 94^{\circ} \mathrm{C} ; 30 \mathrm{sec}, 58^{\circ} \mathrm{C} ; 30 \mathrm{sec}, 72^{\circ} \mathrm{C}$; 34 cycles. The sequences for survivin sense and antisense primers were 5'-GAGGCGGGCGGATCACGAGAG-3' and 5'-TGCTAAGGGGCCCACAGGAAGG-3'. Equal amounts of RT-PCR products were loaded on $1.5 \%$ agarose gels, respectively. GAPDH was used as an internal control. The sequences for GAPDH sense and antisense primers were 5'-TGGGGAAGGTGAAGGTCGG-3' and 5'-CTGGAAGATGGTGATGGGA-3'.

In vivo study in tumor-bearing mice. SGC996 cells were harvested, washed, and resuspended in serum-free optimum

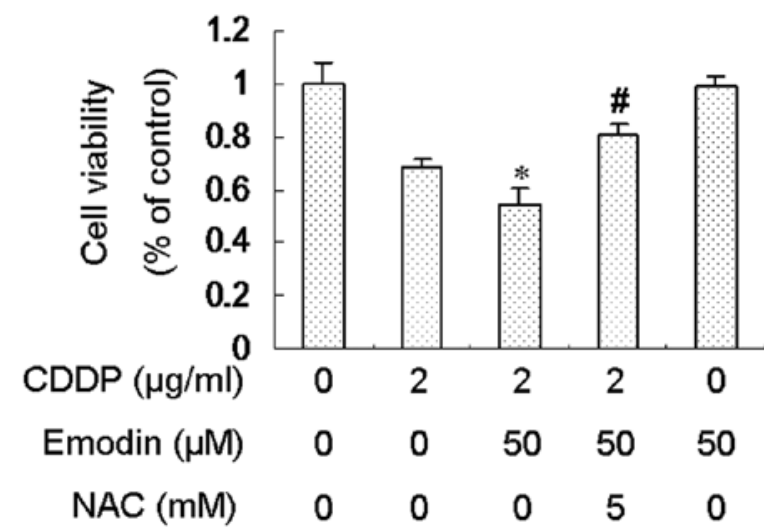

Figure 1. Cell viability in SGC996 cells (MTT). Cells were exposed to emodin alone, CDDP alone, CDDP/emodin co-treatment and co-treatment plus NAC for $24 \mathrm{~h} .{ }^{*} \mathrm{p}<0.05$ vs. CDDP-alone group, ${ }^{*} \mathrm{p}<0.05$ vs. CDDP/ emodin co-treatment group.

medium and then injected subcutaneously into 6-week-old BALB/c-nu/nu mice, with $6 \times 10^{6}$ cells per mouse $(n=8$ mice per group, purchased from Shanghai Experimental Animal Center, Shanghai, China). Three days after inoculation, the tumor-bearing mice were intraperitoneally administered with dissolvent, emodin (50 mg/kg), CDDP (1 mg/kg), emodin/ CDDP every day. The mice were sacrificed after 18 days, and tumor size was measured. Hearts, kidneys and livers of the mice were histologically examined to determine the systemic toxicity.

In situ hybridization for survivin expression in tumors. The sequence of digoxiginin-labeled single-stranded RNA probe for survivin was: 5'-CTCTCGTGATCCGCCCGCCTC-3'. Sections of the tumor tissue were de-paraffined and re-hydrated before incubation with Protease $\mathrm{K}$ at $37^{\circ} \mathrm{C}$ for $10 \mathrm{~min}$. Sections were then washed in $0.1 \mathrm{M}$ Tris-buffered saline/diethyl pyrocarbonat (TBS/DEPC) for $5 \mathrm{~min} \times 3$, incubated with $5 \mathrm{X}$ standard sodium citrate (SSC) solution at RT for $15 \mathrm{~min}$ and incubated with RNA probe sequentially. After $24 \mathrm{~h}$ of hybridization at $37^{\circ} \mathrm{C}$, the sections were washed with graded diluted SSC solutions, all at $37^{\circ} \mathrm{C}$ for $15 \mathrm{~min}$. Then the sections were incubated with anti-digoxiginin antibody (Roche Diagnostics $\mathrm{GmbH}$, Mannheim, Germany) at RT for $3 \mathrm{~h}$, washed with $0.5 \mathrm{M}$ TBS and 0.01 M TBS ( $\mathrm{pH} 9.5$ ). Hybridization signal was visualized by 5-bromo-4-chloro-30-indolyphosphate ptoluidine salt/nitro-blue tetrazolium chloride (BCIP/NBT) (Sigma). Sections were finally counterstained by nuclear fast red.

Statistical analysis. Data are shown as the mean values $\pm \mathrm{SE}$ SPSS11.5 software was used for statistical analysis. ANOVA (analysis of variance) was applied for comparison of the means of two or multiple groups, in which SNK (Student-NewmanKewls) was further used for comparison of each two group. A value of $\mathrm{p}<0.05$ was considered significant.

\section{Results}

Emodin enhances CDDP-induced inhibition of cell viability in SGC996 cells in a ROS-dependent manner. SGC996 cells 
A
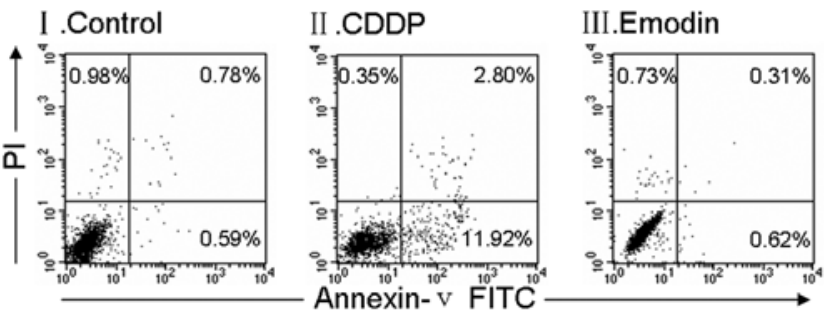

IV .CDDP+Emodin

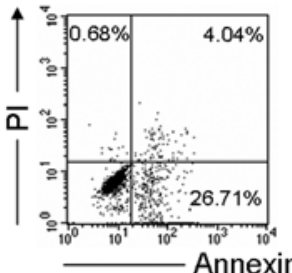

\section{CDDP+Emodin+NAC}

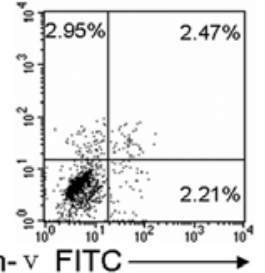

B

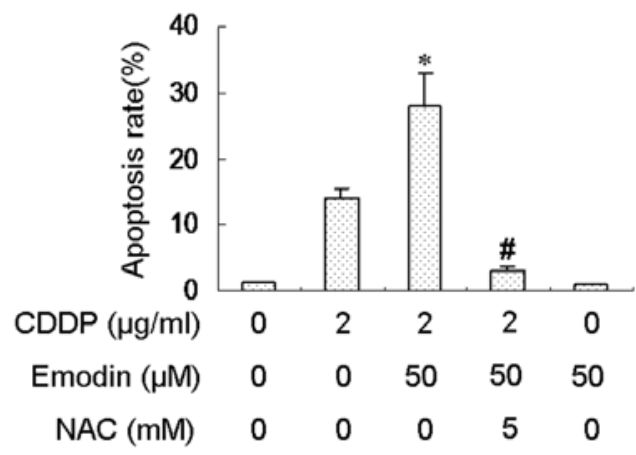

Figure 2. Apoptosis in SGC996 cells. Apoptosis rate analysis using Annexin V/propidium iodide flow cytometry in SGC996 cells treated with CDDP, emodin, the two-drug combination and the two-drug combination plus NAC for $24 \mathrm{~h}$. Density plots (A), bar chart (B). ${ }^{*} \mathrm{p}<0.05$ vs. CDDP-alone group, ${ }^{\# p}<0.05$ vs. CDDP/emodin co-treatment group.

A

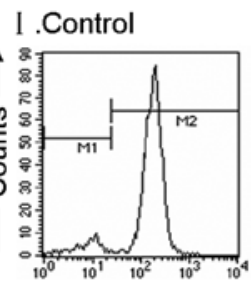

II .CDDP

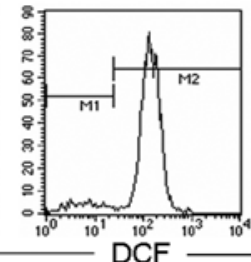

IV.CDDP+Emodin
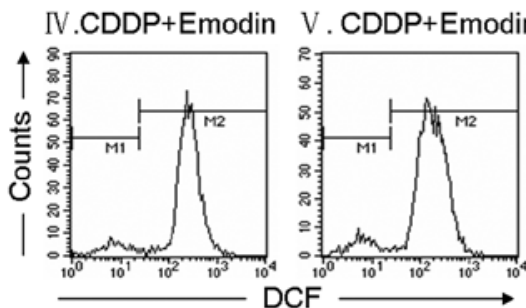

III.Emodin
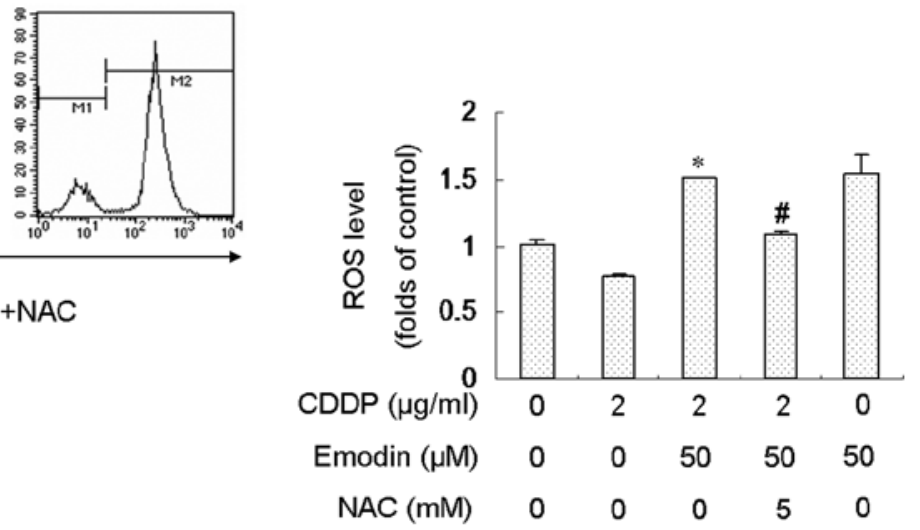

Figure 3. ROS in SGC996 cells (DCF flow cytometry). Cells were exposed to emodin alone, CDDP alone, CDDP/emodin co-treatment and co-treatment plus NAC for 15 min (NAC pre-incubation for 4 h). Histograms (A), bar chart (B). ${ }^{*} \mathrm{p}<0.05$ vs. CDDP-alone group, ${ }^{\#} \mathrm{p}<0.05$ vs. CDDP/emodin co-treatment group.

were treated with antitumor drug CDDP alone or co-treated with $50 \mu \mathrm{M}$ emodin, and cell viability were estimated with MTT assay. CDDP was used at the doses that, when used alone for $24 \mathrm{~h}$, achieved $\sim 30 \%$ repression of viability (CDDP $2 \mu \mathrm{g} / \mathrm{ml})$. Emodin alone $(50 \mu \mathrm{M})$ exerted no or little impact on cancer cell viability. Co-treating cells with emodin at this dose nevertheless caused a significant enhancement of cell viability repression induced by CDDP. The enhancement could be completely reversed by pre-treatment of cells with the antioxidant NAC, suggesting that emodin's effects were dependent on ROS generation (Fig. 1).

Emodin enhances CDDP-induced apoptosis of SGC996 cells in a ROS-dependent manner. To determine whether the viability repression was caused via inhibition of proliferation or enhancement of apoptosis by CDDP alone or CDDP/emodin co-treatment, Annexin V-FITC/PI double labeling flow cytom- etry was conducted. Results showed that cytotoxicity of CDDP was caused predominantly via cell apoptosis. Emodin $(50 \mu \mathrm{M})$ enhanced CDDP-induced apoptosis, while NAC abrogated this enhancement (Fig. 2).

Emodin elicits an immediate elevation of cellular ROS level. To study oxidative impact of emodin on cellular oxidationreduction (redox) state, we measured the cellular ROS level after exposing SGC996 cells to CDDP, emodin or CDDP/ emodin, respectively, for $15 \mathrm{~min}$. We found that emodin alone or in combination with CDDP resulted in an immediate elevation of cellular ROS level, while CDDP treatment alone did not have this effect. Pre-treatment of cells with the antioxidant NAC could completely abolish the elevation of cellular ROS level (Fig. 3). These data indicated that the synergistic effect of emodin on enhancement of CDDP-induced cytotoxicity was related to its generation of ROS in cells. 


\begin{tabular}{|c|c|c|c|c|c|}
\hline Survivin & $=$ & -6 & $\cos$ & $\Rightarrow$ & 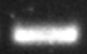 \\
\hline APDH & $=$ & 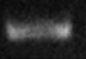 & 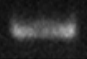 & nest & nes \\
\hline & 0 & 2 & 2 & 2 & 0 \\
\hline $\operatorname{lodin}(\mu)$ & 0 & 0 & 50 & 50 & 50 \\
\hline$A C$ & 0 & 0 & 0 & 5 & \\
\hline
\end{tabular}

Figure 4. Expression of survivin in SGC996 cells. Cells were exposed to CDDP alone, emodin alone, CDDP/emodin co-treatment and co-treatment plus NAC for $24 \mathrm{~h}$ before harvested for RT-PCR.

Emodin/CDDP co-treatment additively downregulates expression of survivin in SGC996 cells in a ROS-dependent manner. It has been reported that overexpression of survivin often render cancer cells a constitutive characteristic of drug resistance, therefore inhibition of its expression may lead to the increase of sensitivity to anticancer drugs. We then questioned whether the observed effects of CDDP/emodin could be correlated with regulating expression of survivin. Results from RT-PCR showed that CDDP alone downregulated expression of survivin, while CDDP/emodin co-treatment resulted in an additive effect on downregulating expression of survivin and NAC reversed this suppression (Fig. 4). These results indicated that the effect of emodin to promote cytotoxicity of CDDP might be achieved, at least partially, via down regulation of survivin, which was again ROS-dependent.

Emodin markedly sensitizes tumor xenografts to CDDP cytotoxicity without displaying obvious systemic toxicity in vivo. The above in vitro experiments showed that multidrug resistant phenotype of SGC996 cells could be partially overcome by using emodin in combination with chemotherapeutic drug CDDP. To verify this effect in vivo and evaluate its systemic efficacy and side effects, SGC996 cells were transplanted into nude mice and the mice were synchronously administered with emodin alone or in combination with CDDP for 18 days. Our results showed that mice exposed to the combinatorial therapy
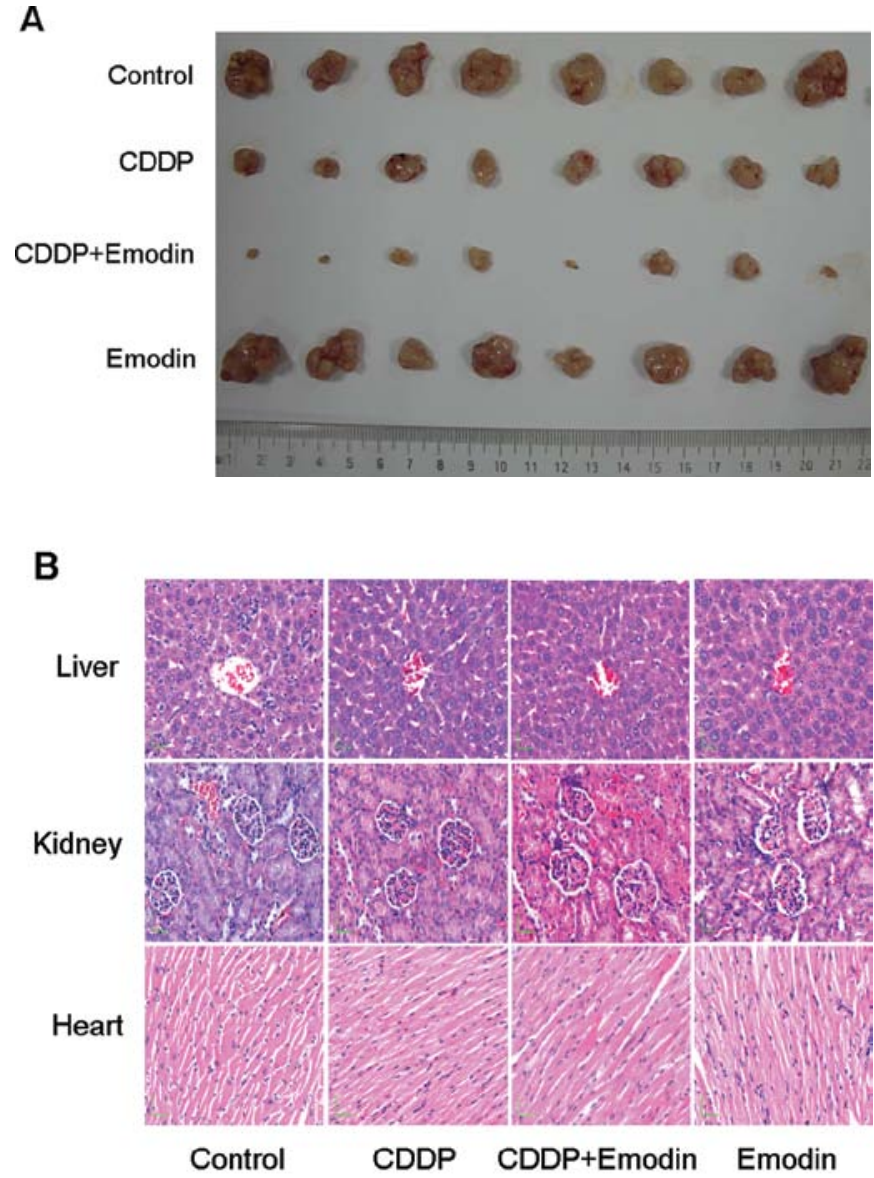

Figure 5. Growth capability of transplanted tumors formed by SGC996 cells The tumor-bearing mice were injected intraperitoneally with dissolvent, $50 \mathrm{mg} / \mathrm{kg}$ emodin alone, $1 \mathrm{mg} / \mathrm{kg}$ CDDP alone and CDDP/emodin coadministration. (A) Photograph of transplanted tumors after the mice were exposed to treatments. (B) Histology of livers, kidneys and hearts of tumor-bearing mice. Scale bar, $30 \mu \mathrm{m}$.

had significantly smaller tumors than mice in other groups (Fig. 5A). While tumor cytotoxicity was strikingly enhanced by the combined treatment, the systemic toxic effects were evaluated by examining the pathological changes of the

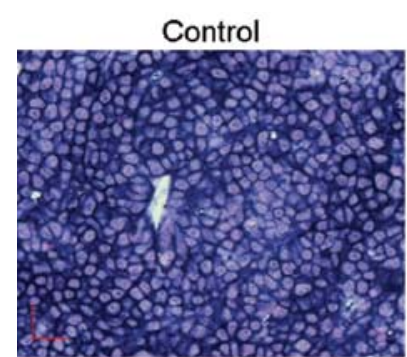

CDDP+Emodin

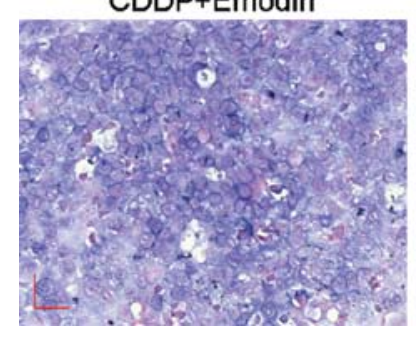

CDDP

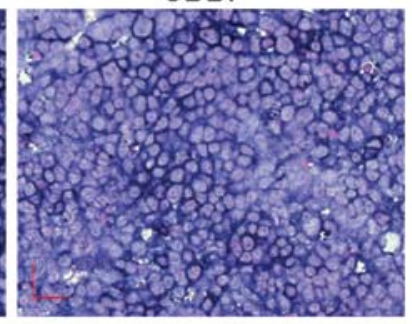

Emodin

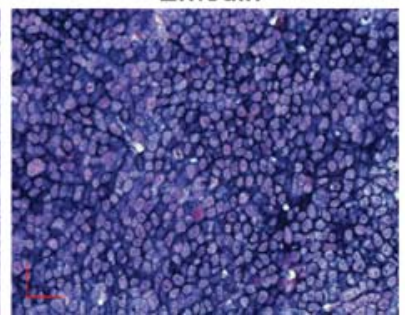

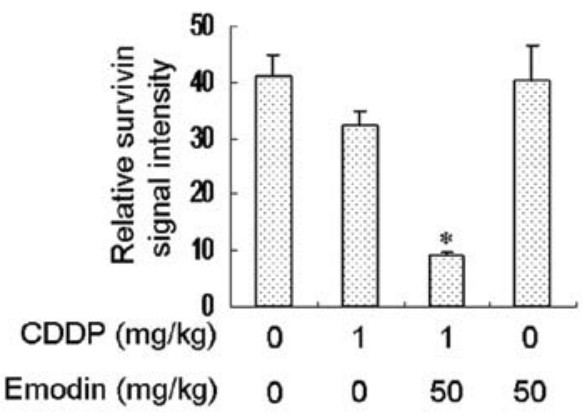

Emodin $(\mathrm{mg} / \mathrm{kg}) \quad 0 \quad 0 \quad 50 \quad 50$

Figure 6. Expression of survivin mRNA in transplanted tumor tissues. The cytoplasmic blue-purple staining represented positive signal for survivin mRNA, and the nucleus were stained by fast red (in situ hybridization). " p<0.05 vs. CDDP-alone group. Scale bar, $30 \mu \mathrm{m}$. 
major organs of mice. No notable differences were observed among these groups (Fig. 5B), suggesting that emodin/CDDP co-treatment had no obvious toxic effects on normal tissues in vivo.

Emodin/CDDP co-treatment represses the expression of survivin in tumors. To ascertain the action of emodin/CDDP co-treatment on survivin expression in vivo, in situ hybridization for survivin mRNA was performed on paraffin-embedded tissue sections of tumors. As shown in Fig. 6, the expression of survivin in tumors was downregulated by CDDP, and in particular, more significantly by emodin/CDDP combined treatment.

\section{Discussion}

For gallbladder cancer at advanced stage, radical resection is quite difficult to achieve to improve the survival rate. Some potent therapeutic strategies which could promote therapeutic effects including chemotherapy are required for better treatment of patients with gallbladder cancer. However, there are no satisfactory chemotherapeutic agents. It is crucial to design new strategies that could enhance the chemotherapeutic efficacy on gallbladder cancer cells. The concept of synergism of cytotoxicity by a relatively non-toxic or moderate toxic compound with a standard drug has opened better anticancer treatment options, even though most of these are still in the experimental stage.

Pharmacological studies have demonstrated that emodin possesses various biological function, such as anti-bacterial (8), anti-inflammatory (9), anti-cancer and a potent inhibitor of the casein kinase 2 (10). Emodin has been reported to exhibit anti-tumor effects in various cancer cells $(11,12)$. Previous studies have demonstrated that emodin inhibits cell growth in several types of tumor cells (13-16) and regulates genes related to the control of cell proliferation, cell apoptosis, oncogenesis and cancer cell invasion and metastasis (17-22). In recent years a reappraisal of different modes of using emodin in the elimination/retardation of growth of tumor cells in combination with chemotherapeutic agents have been done (23). However, co-treatment data were mainly the results from $\mathrm{As}_{2} \mathrm{O}_{3}$ and more drugs need to be recruited for evaluating emodin's synergistic potential, also in different cell systems. We investigated whether the combination of CDDP and emodin could enhance cell growth inhibition more than CDDP alone in human gallbladder cancer cells. We found that the combination of CDDP and emodin treatment induced noticeable tumor cell growth inhibition compared with CDDP alone. Inhibition of cell growth was correlated with increase of apoptotic cell death. In the current study, our results showed emodin could sensitize SGC996 cells to apoptosis induced by CDDP.

The intracellular level of ROS is closely related to the chemosensitivity of cancer cells $(24,25)$. Increase of ROS generation facilitates cytotoxic actions of antitumor drugs, while cells bearing a lower ROS level usually are less responsive to chemotherapy $(24,26,27)$. Moreover, manipulation of redox status of cancer cells to enhance cytotoxicity of drugs has proved to be a potential therapeutic strategy $(24,28)$. For example, L-buthionine sulfoximine, an inhibitor of glutathione production, sensitizes tumor cells to several anticancer drugs, which not only enhances apoptosis in cultured tumor cell lines but also exerts the adjunctive treatment in clinical trials $(29,30)$. Other approaches by either increase of ROS generation or depletion of antioxidant molecules display similar synergistic effects with chemotherapy, photodynamic therapy and radiotherapy (31-33). Emodin is able to generate ROS in a variety of tumor cells $(3,6,34,35)$. In our study, we also found emodin could significantly increase ROS level in SGC996 cells, indicating anticancer actions of emodin might be associated with its ability to generate ROS. That emodin's enhancement of CDDP-induced cytotoxicity in SGC996 cells is apparently dependent on ROS generation was also evidenced by the enhancement of both proliferation-inhibition and apoptosis rendered by cotreatment with the two drugs that can be abolished or attenuated by the antioxidant NAC. The results of the present study have confirmed that emodin exerts synergistic anticancer actions via increase of ROS.

The prosurvival molecule survivin, a member of the inhibitor of apoptosis protein (IAP) family, has been implicated in the control of cell division and apoptosis (36). Survivin's anti-apoptotic function is executed via its ability to prevent caspase activation. Growing evidence suggests that survivin is responsible for drug resistance in cancer cells (37-39). Considering that survivin has been described as an apoptosis inhibitor, we investigated whether emodin enhanced chemosensitivity of SGC996 cells through decreasing survivin levels. Our results demonstrated that CDDP alone could down-regulate survivin expression, and emodin co-treatment with CDDP further repressed the expression of survivin, suggesting that inhibition of survivin by emodin co-treatment is associated with sensitization of gallbladder cancer cells to apoptotic cell death induced by CDDP. Also, we found that antioxidant NAC could reverse down-regulation of survivin expression induced by emodin in combined with CDDP. The result supported our hypothesis that emodin co-treatment decreased survivin levels by ROS generation. Survivin expression is regulated by $N F-\kappa B$ (40-43). We have previously demonstrated that NF- $\kappa \mathrm{B}$ activation was suppressed by cotreatment with emodin and $\mathrm{As}_{2} \mathrm{O}_{3}$ via generation of ROS, suggesting NF- $\kappa \mathrm{B}$ might serve as the target of emodin to mediate down-regulation of survivin when it was coadministered. Of course, survivin expression could be coregulated by factors other than NF- $\kappa \mathrm{B}$ (44). In future work, we will further investigate these important mechanistic details in gallbladder cancer.

In addition to these in vitro results, we found that emodin potentiates the antitumor effects of CDDP in a subcutaneous xenograft gallbladder cancer. The combination treatment resulted in a visibly reduced tumor volume when compared to CDDP single agent treatment. Most importantly, the results in vitro were replayed in vivo, down-regulating the expression of survivin in tumor tissues treated with combination. However, no pathological change in the major organs occurred in the emodinalone treatment group and emodin/CDDP co-treatment group, supporting the conception that ROS manipulation strategy could be selective between cancerous and normal cells, as indicated by an increasing body of reports $(24,45)$. In conclusion, our current findings first showed that emodin potentiates the antitumor effects of CDDP by downregulating survivin levels in gallbladder cancer. Generation of ROS might be playing an 
important role in above process. However, further investigations are necessary to gain more information before clinical use might be possible.

\section{Acknowledgements}

This study was supported by a grant from the Foundation of Shanghai Science and Technology Committee (09411960800).

\section{References}

1. Grobmyer SR, Lieberman MD and Daly JM: Gallbladder cancer in the twentieth century: single institution's experience. World J Surg 28: 47-49, 2004.

2. Qayyum A and Mujtaba I: Effects of chemotherapy on patients with unresectable or metastatic adenocarcinoma of gallbladder. $\mathrm{J}$ Pak Med Assoc 57: 71-74, 2007.

3. Huang XZ, Wang J, Huang C, et al: Emodin enhances cytotoxicity of chemotherapeutic drugs in prostate cancer cells: the mechanisms involve ROS-mediated suppression of multidrug resistance and hypoxia inducible factor-1. Cancer Biol Ther 7: 468-475, 2008

4. Altieri DC: The molecular basis and potential role of survivin in cancer diagnosis and therapy. Trends Mol Med 7: 542-547, 2001.

5. Thomas S and Shah G: Calcitonin induces apoptosis resistance in prostate cancer cell lines against cytotoxic drugs via the Akt/ survivin pathway. Cancer Biol Ther 4: 1226-1233, 2005.

6. Yang J, Li H, Chen YY, et al: Anthraquinones sensitize tumor cells to arsenic cytotoxicity in vitro and in vivo via reactive oxygen species-mediated dual regulation of apoptosis. Free Radic Biol Med 37: 2027-2041, 2004.

7. Jing Y, Yang J, Wang Y, et al: Alteration of subcellular redox equilibrium and the consequent oxidative modification of nuclear factor kappaB are critical for anticancer cytotoxicity by emodin, a reactive oxygen species-producing agent. Free Radic Biol Med 40: 2183-2197, 2006.

8. Wang HH and Chung JG: Emodin-induced inhibition of growth and DNA damage in the Helicobacter pylori. Curr Microbiol 35 262-266, 1997.

9. Chang $\mathrm{CH}$, Lin CC, Yang JJ, Namba T and Hattori M: Antiinflammatory effects of emodin from ventilago leiocarpa. Am J Chin Med 24: 139-142, 1996.

10. Yim H, Lee $\mathrm{YH}$, Lee $\mathrm{CH}$ and Lee SK: Emodin, an anthraquinone derivative isolated from the rhizomes of Rheum palmatum, selectively inhibits the activity of casein kinase II as a competitive inhibitor. Planta Med 65: 9-13, 1999.

11. Cai J, Razzak A, Hering J, et al: Feasibility evaluation of emodin (rhubarb extract) as an inhibitor of pancreatic cancer cell proliferation in vitro. JPEN J Parenter Enteral Nutr 32: 190-196, 2008.

12. Chang CJ, Ashendel CL, Geahlen RL, McLaughlin JL and Waters DJ: Oncogene signal transduction inhibitors from medicinal plants. In Vivo 10: 185-190, 1996.

13. Chen YC, Shen SC, Lee WR, et al: Emodin induces apoptosis in human promyeloleukemic HL-60 cells accompanied by activation of caspase 3 cascade but independent of reactive oxygen species production. Biochem Pharmacol 64: 1713-1724, 2002.

14. Jing $X$, Ueki $N$, Cheng J, Imanishi $H$ and Hada T: Induction of apoptosis in hepatocellular carcinoma cell lines by emodin. Jpn J Cancer Res 93: 874-882, 2002.

15. Srinivas G, Anto RJ, Srinivas P, Vidhyalakshmi S, Senan VP and Karunagaran D: Emodin induces apoptosis of human cervical cancer cells through poly(ADP-ribose) polymerase cleavage and activation of caspase-9. Eur J Pharmacol 473: 117-125, 2003.

16. Zhang L, Lau YK, Xi L, et al: Tyrosine kinase inhibitors, emodin and its derivative repress HER-2/neu-induced cellular transformation and metastasis-associated properties. Oncogene 16: 2855-2863, 1998.

17. Cha TL, Qiu L, Chen CT, Wen Y and Hung MC: Emodin downregulates androgen receptor and inhibits prostate cancer cell growth. Cancer Res 65: 2287-2295, 2005.

18. Huang Q, Shen HM, Shui G, Wenk MR and Ong CN: Emodin inhibits tumor cell adhesion through disruption of the membrane lipid Raft-associated integrin signaling pathway. Cancer Res 66: 5807-5815, 2006.

19. Kim MS, Park MJ, Kim SJ, et al: Emodin suppresses hyaluronic acid-induced MMP-9 secretion and invasion of glioma cells. Int J Oncol 27: 839-846, 2005.
20. Kwak HJ, Park MJ, Park CM, et al: Emodin inhibits vascular endothelial growth factor-A-induced angiogenesis by blocking receptor-2 (KDR/Flk-1) phosphorylation. Int J Cancer 118: 2711-2720, 2006

21. Muto A, Hori M, Sasaki Y, et al: Emodin has a cytotoxic activity against human multiple myeloma as a Janus-activated kinase 2 inhibitor. Mol Cancer Ther 6: 987-994, 2007.

22. Shieh DE, Chen YY, Yen MH, Chiang LC and Lin CC: Emodininduced apoptosis through p53-dependent pathway in human hepatoma cells. Life Sci 74: 2279-2290, 2004.

23. Srinivas G, Babykutty S, Sathiadevan PP and Srinivas P: Molecular mechanism of emodin action: transition from laxative ingredient to an antitumor agent. Med Res Rev 27: 591-608, 2007.

24. Trachootham D, Alexandre J and Huang P: Targeting cancer cells by ROS-mediated mechanisms: a radical therapeutic approach? Nat Rev Drug Discov 8: 579-591, 2009.

25. Pervaiz S and Clement MV: Tumor intracellular redox status and drug resistance - serendipity or a causal relationship? Curr Pharm Des 10: 1969-1977, 2004.

26. Singh N, Azmi S, Sheriff A, Dhawan D and Khanna N: Differential sensitivity of murine myeloid FDC-P1 cells and apoptosis resistant mutant(s) to anticancer drugs. Mutat Res 474: 105-112, 2001.

27. Furusawa S, Kimura E, Kisara S, et al: Mechanism of resistance to oxidative stress in doxorubicin resistant cells. Biol Pharm Bull 24: 474-479, 2001.

28. Renschler MF: The emerging role of reactive oxygen species in cancer therapy. Eur J Cancer 40: 1934-1940, 2004.

29. Bailey HH: L-S,R-buthionine sulfoximine: historical development and clinical issues. Chem Biol Interact 111-112: 239-254, 1998.

30. Maeda H, Hori S, Ohizumi H, et al: Effective treatment of advanced solid tumors by the combination of arsenic trioxide and L-buthionine-sulfoximine. Cell Death Differ 11: 737-746, 2004.

31. Chen Z, Woodburn KW, Shi C, Adelman DC, Rogers C and Simon DI: Photodynamic therapy with motexafin lutetium induces redox-sensitive apoptosis of vascular cells. Arterioscler Thromb Vasc Biol 21: 759-764, 2001.

32. Husbeck B, Peehl DM and Knox SJ: Redox modulation of human prostate carcinoma cells by selenite increases radiation-induced cell killing. Free Radic Biol Med 38: 50-57, 2005.

33. Kitamura K, Minami Y, Yamamoto K, et al: Involvement of CD95-independent caspase 8 activation in arsenic trioxideinduced apoptosis. Leukemia 14: 1743-1750, 2000.

34. Cai J, Niu X, Chen Y, et al: Emodin-induced generation of reactive oxygen species inhibits RhoA activation to sensitize gastric carcinoma cells to anoikis. Neoplasia 10: 41-51, 2008.

35. Yi J, Yang J, He R, et al: Emodin enhances arsenic trioxideinduced apoptosis via generation of reactive oxygen species and inhibition of survival signaling. Cancer Res 64: 108-116, 2004.

36. Ambrosini G, Adida C and Altieri DC: A novel anti-apoptosis gene, survivin, expressed in cancer and lymphoma. Nat Med 3: 917-921, 1997.

37. Altieri DC: Validating survivin as a cancer therapeutic target. Nat Rev Cancer 3: 46-54, 2003.

38. Li F: Role of survivin and its splice variants in tumorigenesis. $\mathrm{Br}$ J Cancer 92: 212-216, 2005

39. Zaffaroni N, Pennati M and Daidone MG: Survivin as a target for new anticancer interventions. J Cell Mol Med 9: 360-372, 2005.

40. Mitsiades CS, Mitsiades N, Poulaki V, et al: Activation of NF-kappaB and upregulation of intracellular anti-apoptotic proteins via the IGF-1/Akt signaling in human multiple myeloma cells: therapeutic implications. Oncogene 21: 5673-5683, 2002.

41. Yamamoto Y and Gaynor RB: Therapeutic potential of inhibition of the NF-kappaB pathway in the treatment of inflammation and cancer. J Clin Invest 107: 135-142, 2001.

42. Zhu L, Fukuda S, Cordis G, Das DK and Maulik N: Antiapoptotic protein survivin plays a significant role in tubular morphogenesis of human coronary arteriolar endothelial cells by hypoxic preconditioning. FEBS Lett 508: 369-374, 2001.

43. Qi $\mathrm{H}$ and Ohh M: The von Hippel-Lindau tumor suppressor protein sensitizes renal cell carcinoma cells to tumor necrosis factor-induced cytotoxicity by suppressing the nuclear factor-kappaB-dependent antiapoptotic pathway. Cancer Res 63: 7076-7080, 2003.

44. Reed JC: The Survivin saga goes in vivo. J Clin Invest 108: 965-969, 2001.

45. Wang $\mathrm{J}$ and Yi J: Cancer cell killing via ROS: to increase or decrease, that is the question. Cancer Biol Ther 7: 1875-1884, 2008. 